\title{
Hypotension During Haemodialysis: Aetiology, Risk Factors and Outcome
}

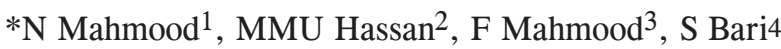

\begin{abstract}
Background: Hypotension during hemodialysis is common. In some patients, the development of hypotension necessitates intravenous fluid replacement before patients are able to safely leave the dialysis unit. Intradialytic hypotension may reduce the efficacy of the dialysis procedure and contributes to the excessive morbidity and mortality that is associated with hemodialysis.

Aims: The aim of the study is to identify the patients who develops intradialytic hypotension and find out the aetiology, risk factors and outcome to reduce the mortality and morbidity of those patients.

Methodology: This Cross sectional descriptive type of observational study was conducted in the Haemodialysis Unit of Anwer Khan Modern Medical College Hospital over the period from January 2015 to December 2016. The change in systolic pressure during haemodialysis was reviewed for ten consecutive haemodialysis in all the patients. The patients with chronic renal failure who were on regular dialysis were enrolled. Patients with sepsis, hemoglobin level less than $9 \mathrm{~g} / \mathrm{dL}$, low cardiac output, acute coronary syndrome, significant valvular lesion or body weight below the dry weight of the patient were excluded from the study.

Result: Ninetythree patients were included in the study. Among them male 53(56.98\%) and female

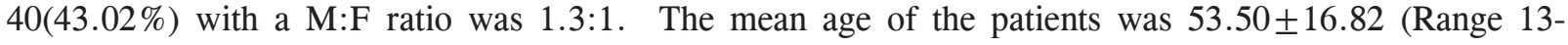
90). We found that $28 \%$ (26) of the patients developed intradialytic hypotension at least once in consecutive ten dialysis. Out of them 14 were female and 12 patients were male. All of the patients recovered from the incident after adequate management and stoppage of the ultrafiltrate volume, convert the machine to SLED(Sustained low efficacy dialysis) mode and hold antihypertensive drugs morning dose on the day of haemodialysis and treatment of the cause.

Conclusion: As a result of increasing age of the patients receiving hemodialysis and the increasing incidence of diabetic nephropathy, hypotension will become a greater problem in future.
\end{abstract}

Key Words: Hypotension, Haemodialysis

\section{Introduction}

Hypotension during hemodialysis is common. In necessitates intravenous fluid replacement before some patients, the development of hypotension patients are able to safely leave the dialysis unit.

\author{
${ }^{1}$ Dr. Nazneen Mahmood, Associate Professor and Head, Department of Nephrology, Anwer Khan Modern \\ Medical College \& Hospital \\ ${ }_{2}^{2}$ Prof (Dr.) Md. Mahtab Uddin Hassan, Ex-Professor and Head, Department of Medicine Anwer Khan Modern \\ Medical College \& Hospital \\ ${ }^{3}$ Dr. Farhana Mahmood, Assistant Professor, Department of Medicine, Chattagram Maa O Shishu Hospital \\ Medical College \\ ${ }^{4}$ Dr. Sabrina Bari, Medical Officer, Department of Nephrology, Anwer Khan Modern Medical College \& Hospital \\ *Corresponding Author
}

Date of submission: 27.12.2016, Date of acceptance: 11.02.2017

AKMMC J 2017; 8(2) : 89-93 
Intradialytic hypotension may reduce the efficacy of the dialysis procedure and contributes to the excessive morbidity and mortality that is associated with hemodialysis.

Symptomatic hypotension during (or immediately following) hemodialysis complicates 5 to 30 percent of all dialysis treatments ${ }^{1,2}$. In one study that included 44,801 dialysis treatments in 1137 patients, 75 percent of patients had at least one episode of intradialytic hypotension 1 . In some patients, more than 50 percent of treatments are complicated by intradialytic hypotension ${ }^{3}$.

Intradialytic hypotension (IDH) remains to be a major complication of hemodialysis. It occurs in nearly $25 \%$ of dialysis sessions ${ }^{4}$ and often requires aggressive resuscitative measures and sometimes premature termination of hemodialysis. It is also a significant independent factor affecting mortality in hemodialysis patients ${ }^{4,5}$.

Despite the advances of machines with ultrafiltration control devices, modifying dialysate composition, temperature control, correction of nutritional deficiencies and treatment of anemia with erythropoietin therapy, many patients still have episodes of intradialytic hypotension. Among other factors, the major pathophysiology of these episodes is the removal of large volume of blood water and solutes over a short period of time, overwhelming normal compensatory mechanisms, which include plasma refilling and reduction of venous capacity (due to reduction of pressure transmission to veins).

In some patients, a seemingly paradoxical and inappropriate reduction of sympathetic tone may occur, causing reduction of arteriolar resistance, decreased transmission of pressure to veins with corresponding increase in venous capacity. Increased sequestration of blood in veins under conditions of hypovolemia reduces cardiac filling, cardiac output and ultimately blood pressure. Hypotensive episodes during hemodialysis in patients with end stage renal disease in absence of inadequate maintenance of the plasma volume, pre-existence of cardiovascular disease, or autonomic nervous system dysfunction are accompanied by increased plasma concentrations of the end-products of nitric oxide metabolism (above the expected levels, based on the reduction of $\left.u^{2} a^{6}\right)$.
The characteristics of patients receiving chronic hemodialysis in the Bangladesh has continuously changed over the past three decades. Today the patients are older, and the incidence of diabetic nephropathy has markedly increased. Hypotension during hemodialysis is the most common and bothersome complication of chronic hemodialysis. The demographics of those patients who experience hypotension during dialysis has not been previously identified. The aim of the study is to identify the patients who develops intradialytic hypotension and find out the aetiology, risk factors and outcome to reduce the mortality and morbidity of those patients. We sought to establish if there were fewer hypotensive events when using convective therapies such as haemodiafiltration(HDF) in comparison to conventional haemodialysis(HD) therapy.

\section{Materials and Methods}

This Cross sectional descriptive type of observational study was conducted in the Haemodialysis Unit of Anwer Khan Modern Medical College Hospital over the period from January 2015 to December 2016. The change in systolic pressure during haemodialysis was reviewed for ten consecutive haemodialysis in all the patients. All patients included in the study provided informed written consent. The patients with chronic renal failure who were on regular dialysis were enrolled.

Patients with sepsis, hemoglobin level less than 9 $\mathrm{g} / \mathrm{dL}$, low cardiac output, acute coronary syndrome, significant valvular lesion or body weight below the dry weight of the patient were excluded from the study. All eligible patients were subjected to full history taking and clinical examination. Serum Urea and Creatinine were measured. Glomerular filtration rate (GFR) was calculated using Cockcroft's and Gault equation.

Dialysis was done via AV fistula or dialysis catheter. Nipro machines were used with ultrafiltration volume control and polysulfone, Fresenius F6 filters. Temperature of dialysate was kept at $36{ }^{\circ} \mathrm{C}$. Blood pump was kept between 200 and $220 \mathrm{ml} / \mathrm{min}$ except during the hypotensive episodes during which the blood pump was decreased to only $150 \mathrm{ml} / \mathrm{min}$ and not less to insure 
adequate dialysis session. Dialysate flow was 500 $\mathrm{ml} / \mathrm{min}$. All dialysis sessions lasted around $4 \mathrm{~h}$.

BP was recorded using standard sphygmomanometer every $30 \mathrm{~min}$ in supine position. Each time the mean of 3 measurements was recorded.

After dialysis, myocardial ischemia was assessed using ECG. Statistical analysis was done using Statistical Package for Social Sciences (SPSS) software, release 16.0.0 for Windows ${ }^{\mathrm{TM}}$ (SPSS Inc., Chicago, Illinois). Categorical variables are described as frequency (n) and percentage (\%). Quantitative variables are described as mean \pm standard deviation (SD) whenever parametric.

\section{Operational Definition}

\section{Intradialytic Hypotension}

There is no generally accepted definition of intradialytic hypotension. Kidney Disease Outcomes Quality Initiative (K/DOQI) and European Best Practice Guidelines define intradialytic hypotension as the presence of a decrease in systolic blood pressure $>20 \mathrm{~mm}$ of $\mathrm{Hg}$ or a decrease in mean arterial pressure by $10 \mathrm{~mm}$ of $\mathrm{Hg}$, which is associated with clinical events and need for nursing interventions ${ }^{7}$.

\section{Result}

Ninetythree patients were included in the study. Among them male 53(56.98\%) and female $40(43.02 \%)$ with a M:F ratio was $1.3: 1$. The mean age of the patients was $53.50 \pm 16.82$ (Range 1390). Their baseline characteristics were listed in Table-I.Out of 93 patients, 7 started dialysis with $\mathrm{AV}$ fistula and rest of the patients(85) started through femoral catheter and then AV fistula after a month.

We found that $28 \%(26)$ of the patients developed intradialytic hypotension at least once in consecutive ten dialysis. Out of them 14 were female and 12 patients were male.

The risk factors for developing hypotention during haemodialysis were listed in Table II.
All of the patients recovered from the incident after adequate management and stoppage of the ultrafiltrate volume, convert the machine to SLED(Sustained low efficacy dialysis) mode and hold antihypertensive drugs morning dose on the day of haemodialysis and treatment of the cause.

Table- I: Baseline characteristics of the study group $(\mathrm{N}=93)$

\begin{tabular}{ll}
\hline Parameter & Value [N (\%) or mean + SD] \\
\hline Age (Years) & $53.50+16.82$ \\
Weight (KG) & $56+15$ \\
Male & $53(56.98 \%)$ \\
Female & $40(43.02 \%)$ \\
\hline Clinical & \\
\hline Smokers & $49(92.45 \%)$ \\
Hypertension & $85(91.39 \%)$ \\
Diabetes Mellitus & $39(41.93 \%)$ \\
History of Chest Pain & $16(17.20 \%)$ \\
Family, history of IHD & $21(22.58 \%)$ \\
Autonomic Neuropathy & $39(41.93 \%)$ \\
A V Fistula & $7(7.52 \%)$ \\
Duration of Hemodialysis (Years) & $2.2+1.6$ \\
\hline ECG and Echo Cardiogram & \\
\hline Arrhythmia in ECG & $5(5.37 \%)$ \\
L V H in ECG & $82(96.47 \%)$ \\
EF (\%) & $62.3+7.3$ \\
\hline Grades of Albuminuria & \\
\hline (+) & $3(3.23 \%)$ \\
(+ +) & $78(83.87 \%)$ \\
(++ +) & $12(12.90 \%)$ \\
Urine Specific Gravity & $1008+2.3$ \\
\hline Labs & \\
\hline HS (gm/dl) & $10.4+0.73$ \\
Hct (\%) & $30.6+1.9$ \\
TLC (103 cells/cumm) & $6.1+2.1$ \\
FBS (mg/dl) & $6.2+1.5$ \\
PPBS (mg/dl) & $9.8+2.3$ \\
HbA1c (\%) & $6+0.59$ \\
Serum Triglyceride (mg/dl) & $109.7+43$ \\
Serum Cholesterol (mg/dl) & $151+60$ \\
\hline & $129.8+29.7$ \\
\hline Serum Urea (mg/dl) & \\
\hline
\end{tabular}

EF- Ejection Fraction $\mathrm{Hb}$ - Haemoglobin Hct Haematocrit TLC -Total Leukocyte Count

PPBS - Post Prandial Blood Sugar 
Table-II : Risk factors for Intradialytic Hypotension

\begin{tabular}{cl}
\hline SL No & Risk Factors \\
\hline 1. & Older age group \\
2. & Female Sex \\
3. & Diabetic autonomic neuropathy \\
4. & Intake of anti hypertensive drugs before haemodialysis \\
5. & Large ultra filtrate volume removal \\
6. & Peripheral vascular diseases \\
\hline
\end{tabular}

\section{Discussion}

Intradialytic hypotension(IDH) is a major complication of haemodialysis. Two to four liters of fluid needs to be removed during a regular session, equivalent to $40-80 \%$ of the blood volume. It is therefore not surprising that hypotension occurs so often. Although many factors- patient or treatment related- play a role, a reduction of blood volume is crucial in its pathogenesis ${ }^{1}$. In our study we found 28\%(26) patients developed hypotension during haemodialysis. In the study of James J Flynnll the incidence of patients decreases of at least $40 \mathrm{~mm}$ of systolic pressure, was over $50 \%$ of their treatments was $63 \%$ and the incidence of patients having symptoms from hypotension during haemodialysis was $25 \%$ which was near to our study ${ }^{8}$.

But in the study of Randa Aly Soliman et al $70 \%$ of the patients developed intradialytic hypotension which was not near to our study ${ }^{9}$. Kathleen E Hill et al, 2015 showed that $13.8 \%$ dialysis treatments has at least one episode of hypotension and pre-existing $\mathrm{CAD}$ (Coronary artery disease) increased the odds of hypotension by $77 \% 10$.

Analysis of the dialysis population shows that the age of the patients is a primary factor leading to hypotension. Diabetic nephropathy is identified as a second risk factor for hypotension. In the group of patients (28\%) who have hypotension during hemodialysis with clinical symptoms, all had hypertension requiring therapy prior to initiating hemodialysis. Hypertension may be a predisposing factor to the development of hypotension. Females tended to have more hypotension during dialysis than males.
The reasons for hypotension in dialysis patients, although traditionally assumed to be related to large volumes of fluid removal in a relatively short time, can also be related to cardiac pathology and diastolic dysfunction and the incidence of sudden cardiac death is high in HD patients ${ }^{11}$.

\section{Conclusion}

The incidence of hypotension is thought to be related to pre-existing coronary artery disease(CAD), which represents a considerable challenge for dialysis staff. It is imperative, therefore, that those patients at high risk of hypotension are identified early and appropriately managed with comprehensive fluid balance control and high frequency monitoring during the dialysis therapy. As a result of increasing age of the patients receiving hemodialysis and the increasing incidence of diabetic nephropathy, hypotension will become a greater problem in future.

\section{Acknowledgement}

The researcher would like to acknowledge the valuable support received from the nursing staff of the Haemodialysis unit of AKMMCH and also acknowledge the participants and their families willingness to be involved, which allowed us to conduct the research. The researcher would also like to acknowledge Mr. Zakir Hossain, Librarian, AKMMCH.

\section{Conflict of interest: no.}




\section{References}

1. Sands JJ, Usvyat LA, Sullivan T, et al. Intradialytic hypotension: frequency, sources of variation and correlation with clinical outcome. Haemodial Int 2014; 18: 415.

2. Reilly RF. Attending rounds: A patient with intradialytic hypotension. Clin J Am Soc Nephrol 2014; 9: 798.

3. Santos SF, Peixoto AJ, Perazella MA. How should we manage adverse intradialytic blood pressure changes? Adv Chronic Kidney Dis 2012; 19: 158 .

4. G Ligtenberg, PJ Blakestijn,F Boomsma, HA Koomans. No change in automatic function tests during uncomplicated haemodialysis. Nephrol Dial Transplant. 1996; 11(4): 651656.

5. T Shoji, Y Tsubakihara, M Fujii, E Imai. Haemodialysis associated hypotension as an independent risk factor for two year mortality in hemodialysis patients. Kidney Int. 2004; 66(3):1212-1220.

6. F Madore, L Prud'homme, JS Austin, G Blaise, M Francoeur, M Leveille, et al. Impact of nitric oxide on blood pressure in haemodialysis patients. Am J Kidney Dis. 1996; 30(5): 665671.
7. Kooma J, Basci A, Pizzarelli F et al. EBPG guideline on haemodynamic instability. Nephrol Dial Transplant 2007; 22 suppl 2: ii 22.

8. James J Flynnll. Factors contributing to hypotension during haemodialysis. Geriatric Nephrol Urol. 1996; 6(2): 99-104. Doi: 10.1007/ BF00451113.

9. Randa Aly Soliman, Mohammed Fawzy, Hussein Kandil, Alia Abd el Fattah. Assessment of hypotension during dialysis as a manifestation of myocardial ischemia in patients with chronic renal failure. Egyptian J of CC Medicine. 2014; 2(1):13-18.

10. Kathleen E Hill et al. Dialysis-associated hypotension in haemodiafiltration versus conventional haemodialysis. Renal Society of Australasia Journal. 2015; 11(1): 26-31.

11. Daugirdas JT. Pathophysiology of dialysis hypotension: An update. American Journal of Kidney Diseases. 2001; 38(4): S11-S7. 